\title{
Electrorheological Properties of Suspensions of Polypyrrole Ribbon Particles in Silicone Oil
}

\author{
Shawqi Almajdalawi, Vladimir Pavlinek, Qilin Cheng, Petr Saha, Miroslav \\ Mrlik and Martin Stenicka
}

Centre of Polymer Systems, Polymer Centre, Tomas Bata University in Zlin, nam. T. G. Masaryka 5555, 76001 Zlin, Czech Republic

\begin{abstract}
A new polypyrrole (PPy) ribbon particles structure has been synthesized by solution chemistry method (oxidative polymerization) in the presence of surfactant cetyltrimethylammonium bromide $(\mathrm{CTAB})$ with oxidizing agent ammonium persulfate (APS). The resulted polymer was treated with ammonium hydroxide $\left(\mathrm{NH}_{4} \mathrm{OH}\right)$ several times to obtain different sample conductivity. PPy morphology was characterized by SEM. The suspensions were prepared by dispersing PPy with different conductivities in silicone oil. The electrorheological effect (ER) for the suspensions was examined under various electric fields using a rotational rheometer. It was found that suspension of only 3 wt. \% of PPy ribbon particles showed large ER effect providing yield stress of $256 \mathrm{~Pa}$ at $3 \mathrm{kV} \cdot \mathrm{mm}^{-1}$.
\end{abstract}

Keywords: Polypyrrole ribbon particles, Electrorheology, Conductivity.

PACS: 47.57.ef, 75.50.Bb, 81.40.Rs, 83.60.Fg, 83.60.La, 83.80.Gv

\section{INTRODUCTION}

An electrorheological (ER) fluid is an electroresponsive system which rheological and viscoelastic properties are changed quickly upon application of an external electric field. ER fluids usually consist of two phases; polarizable particles suspended in nonconducting continuous phase. In the absence of electric field ER fluids show usually Newtonian behaviour while in the presence of electric field due to formation of chain like particle structures they show Bingham behaviour with yield stress. When electric field is removed suspensions return to their original liquid like character. Reversible change of rheological properties (viscosity, yield stress) of ER fluids offer potential applications in electric clutches or brakes [1], shock absorbers [2], valves and others [3]. However, there are still some difficulties in practical engineering especially due to insufficient performance of the fluids studied so far.

The ER effect has been observed and investigated in many kinds of suspensions including both organic and inorganic materials [4-9]. However, conducting polymers e.g. polypyrrole (PPy) have attracted considerable attention as suitable materials for electrorheological applications [10]. Besides neat PPy, core-shell structures containing PPy in shell layer and various materials forming the core were investigated [11-14]. PPy 
with silica and methylcellulose nanocomposite suspensions have shown that the ER effect increases with the increase in the silica/pyrrole ratio and the yield stress showed a maximum with increasing methylcellulose amount, followed by a decrease [15].

The ER effect is also significantly influenced by size and shape of the particles. Larger aspect ratio (fibers, rods, ellipsoids) particles show larger ER effect than spherical ones $[6,16]$. Therefore, PPy ribbon particles as a novel material for preparation of ER suspensions were synthetised and role of shape and dielectric properties of PPy particles were investigated.

\section{EXPERIMENTAL}

\section{Chemicals}

Pyrrole (Py, 98\%) was distilled once under reduced pressure before the use. Ammonium persulfate (APS, 98\%) obtained from Sigma-Aldrich (USA) and cetyltrimethylammonium bromide $(\mathrm{CTAB}, 99 \%)$ and ammonium hydroxide $\left(\mathrm{NH}_{4} \mathrm{OH}\right.$, 25-27\%) obtained from LACH-NER (Czech Republic) were used without further treatment.

\section{Synthesis of Material}

PPy ribbon particles were synthesized according to the procedure [17], using pure distilled Py in aqueous media at temperature $(0-5)^{\circ} \mathrm{C}$. After addition of CTAP and APS, the solution was left to polymerize under this temperature with stirring for $24 \mathrm{~h}$. The precipitate was filtered and washed with distilled water and ethanol several times and then dried at $80^{\circ} \mathrm{C}$ for $24 \mathrm{~h}$. Later synthesized PPy ribbon particles were treated with $\mathrm{NH}_{4} \mathrm{OH}$ for $4 \mathrm{~h}$ to reduce polymer conductivity. Sample (PPy1) was treated with $\mathrm{NH}_{4} \mathrm{OH}$ once while the sample (PPy2) was treated twice. Thus two sample particles varying in conductivity (dielectric properties) were obtained.

\section{Preparation and ER Measurements of PPy Ribbon Particle Suspensions}

PPy ribbon particle suspensions ( 3 wt. \%) were prepared by mixing particle samples with corresponding amount of silicone oil (Lukosiol M200, Chemical Works Kolin,

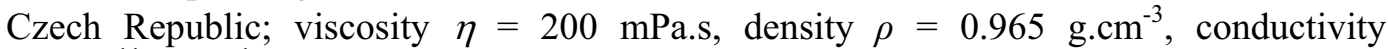
$\left.\sigma \approx 10^{-11} \mathrm{~S} . \mathrm{cm}^{-1}\right)$. Steady and oscillatory shear experiments were performed using Bohlin Gemini rheometer (Malvern Instruments, UK), with parallel plates of $40 \mathrm{~mm}$ in diameters and separated by gap of $0.5 \mathrm{~mm}$. Shear rate ranged from 0.5 to $200 \mathrm{~s}^{-1}$. Viscoelastic properties were investigated in frequency range 0.1-10 Hz. Electric field strength of 0-3 kV.mm ${ }^{-1}$ was applied in all experiments. Prior to each measurement the suspensions were sheared in order to destroy the sample structures developed in electric field for $100 \mathrm{~s}$ at shear rate $20 \mathrm{~s}^{-1}$. The temperature was kept constant at $25^{\circ} \mathrm{C}$ for all experiments. 
Dielectric properties of suspensions were measured using Impediance Material Analyzer Agilent 4294-A, (Agilent, Japan) in frequency range $50 \mathrm{~Hz}-1 \mathrm{MHz}$.

\section{RESULTS AND DISCUSSION}

Figure 1 shows the scanning electron microscopy (SEM) image of the synthesized PPy. The morphology of the sample particles is a network of nanowires forming ribbons. Elemental nanowires should be with the range $25-85 \mathrm{~nm}$ [17].

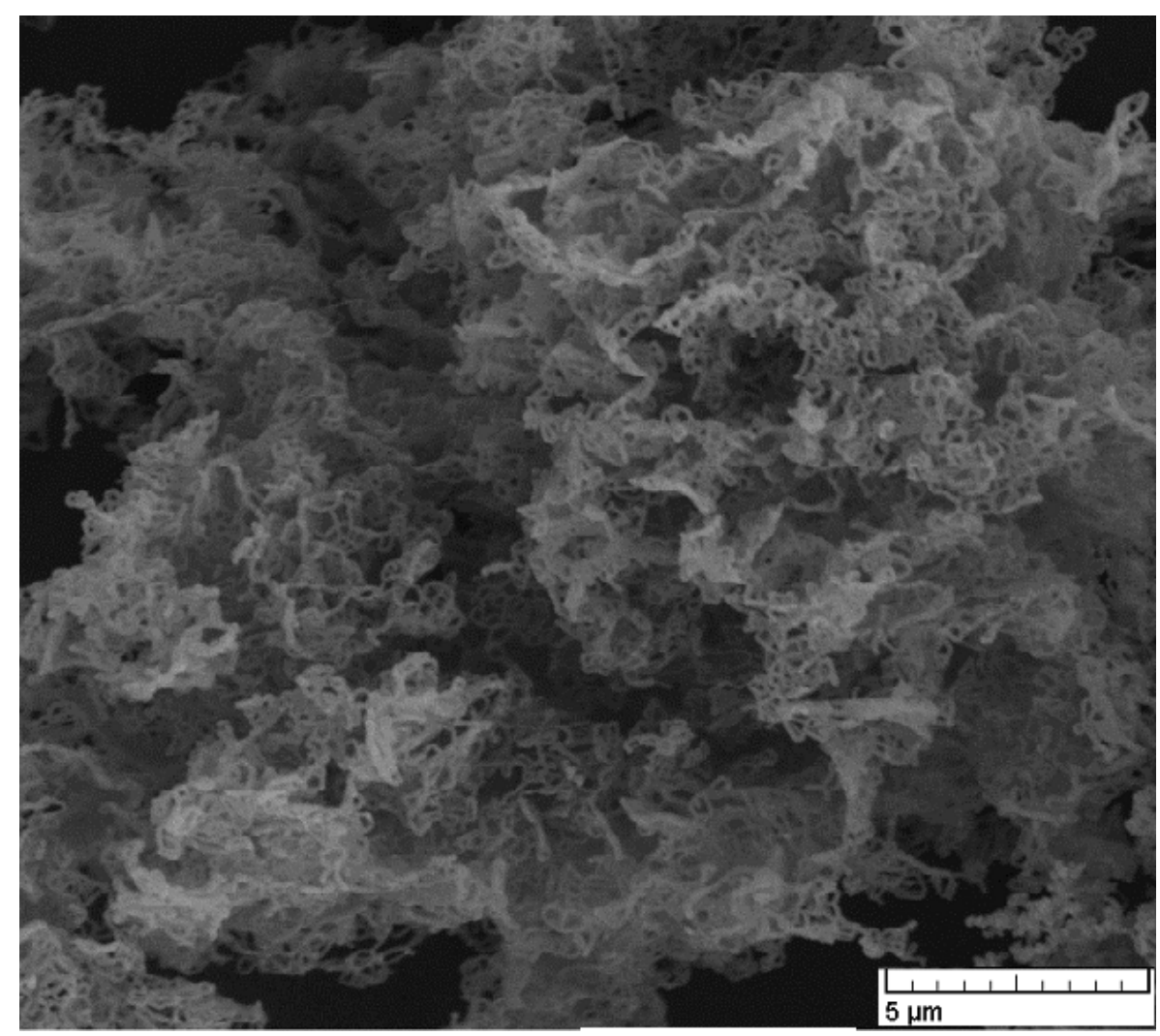

FIGURE 1. SEM picture of polypyrrole particle structure.

Figure 2 shows dependence of shear stress on shear rate. ER fluids in the absence of electric field behave as Newtonian fluids, i.e. the dependence of shear stress on shear rate is linear. However, upon application of electric field particles become polarized and aligned in organized chains or columns which strengthen the whole structure of the suspension. The shear stress increases quickly with increasing electric field strength, and 
the fluid shows non- Newtonian behavior. As can be seen, suspensions of samples PPy1 and PPy 2 showed nearly Newtonian behavior and exhibit no yield stress in the absence of electric field with only slight differences between PPy1 and PPy2. However, when the electric field was applied suspensions behaved as non-Newtonian fluids and exhibited remarkable yield stress for both samples. The yield stress value increased with increasing electric field strength. Suspension of sample PPy2 shows higher yield stress than PPy1. Different ER performance of samples PPy1 and PPy2 is caused by the variation in electric properties and will be discussed further. Suspension of PPy2 sample showed very large ER effect providing yield stress value of $256 \mathrm{~Pa}$ at $3 \mathrm{kV} \cdot \mathrm{mm}^{-1}$. Moreover, shear stress exhibits a plateau region followed by a decrease especially at highest electric field strengths. This phenomenon appears due to the competition between electrostatic and hydrodynamic forces and is commonly observed in suspensions of conductive polymers [18].

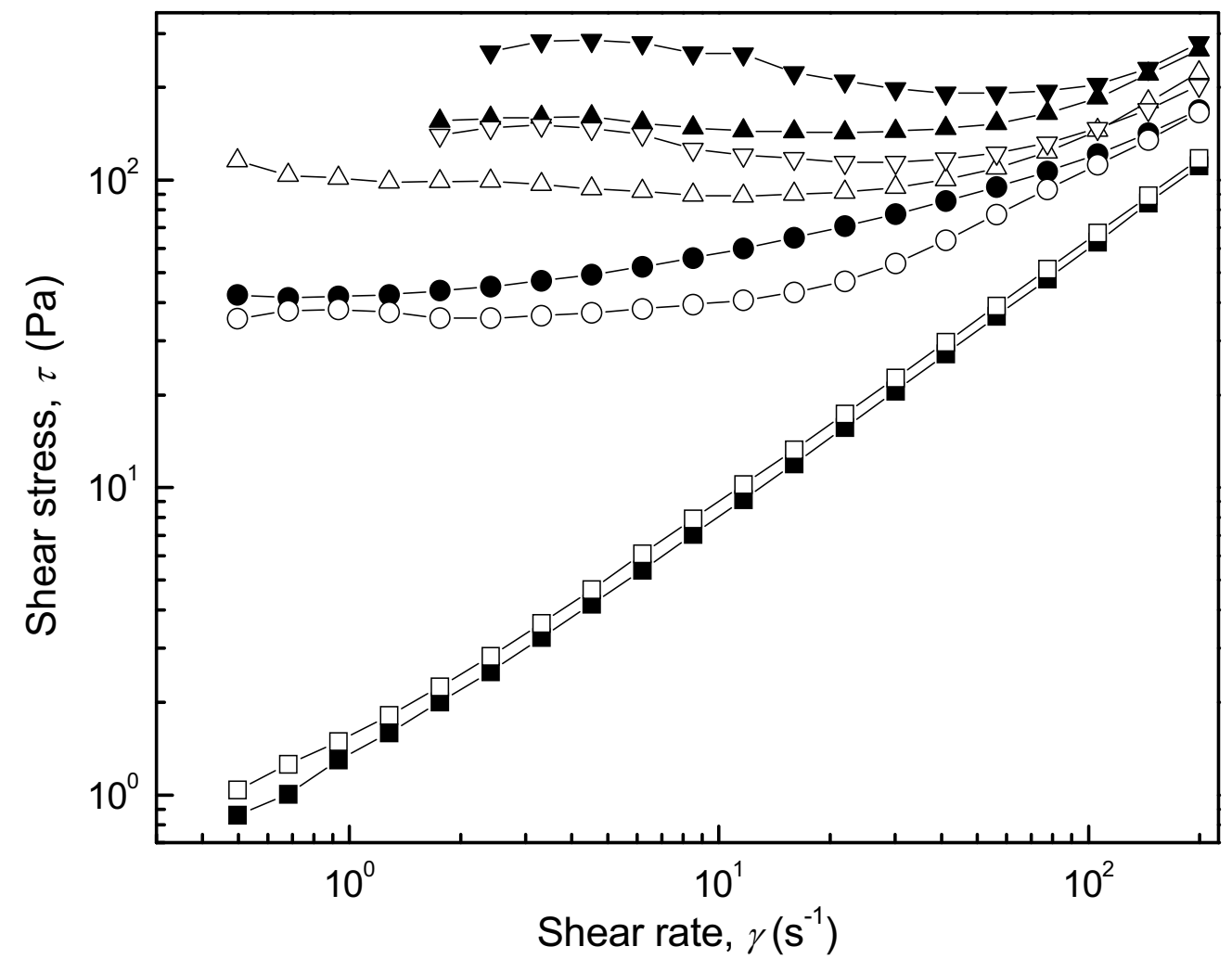

FIGURE 2. Shear stress, $\tau$, vs. shear rate, $\gamma$, dependence for 3 wt. \% polypyrrole ribbon particles PPy1 (open) and PPy2 (solid) suspensions. Electric field strengths E $\left(\mathrm{kV} . \mathrm{mm}^{-1}\right):(\square, \mathbf{\square})$ 0.0, $(\bigcirc, \boldsymbol{\bullet}) 1.0,(\triangle, \mathbf{\Delta}) 2.0,(\nabla, \boldsymbol{\nabla}) 3.0$. 
Figure 3 shows the dynamic yield stress as a function of electric field strength for PPy 1 and PPy 2 suspensions. The linear log-log plot of $\tau_{0}$ vs. $E$ obeys the power law $\tau_{0}=q E^{\alpha}$, where the response of particle polarizability to the electric field strength $\alpha=1.63$ (PPy1) and $\alpha=1.73$ (PPy2) corresponds to the theoretical predictions for well developed ER structures $(\alpha=1.5)$ [19]. Higher $q$ value for suspension of PPy2 sample (1.45) corresponds to higher stiffness of generated ER structures in comparison to suspension of PPy1 sample (1.13).

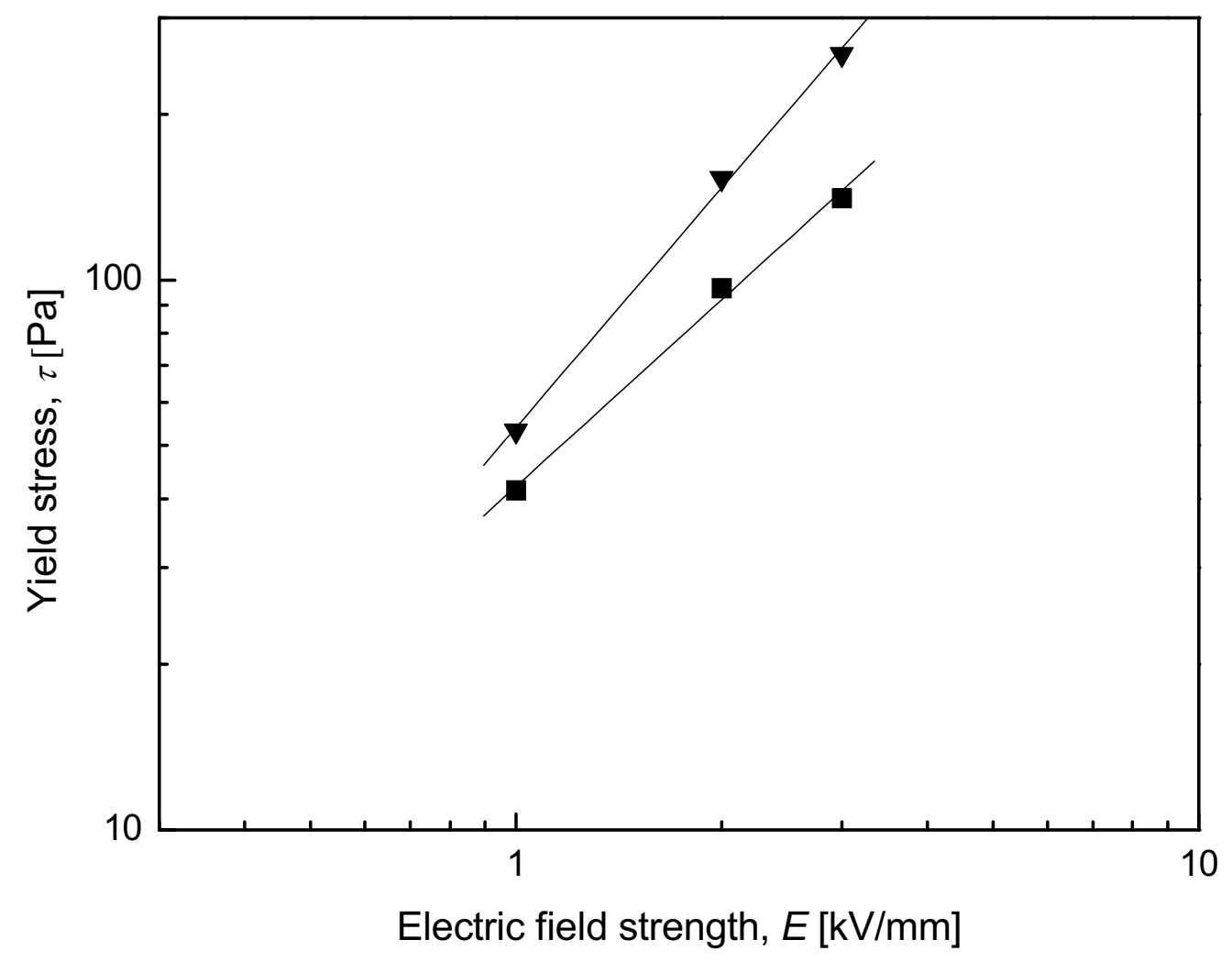

FIGURE 3. Dependence of the yield stress, $\tau$, on electric field strength, $E$, for 3 wt. \% polypyrrole ribbon particles PPy1 (ם) and PPy2 ( $)$ suspensions. 
Dependence of viscosity on shear rate is shown in Figure 4 for both samples at various electric field strengths. Viscosity showed a significant increase with electric field strength especially at lower shear rates. As the shear rate increases, viscosity decreases rapidly. At higher shear rates hydrodynamic forces overcome electrostatic ones and viscosity of suspensions in the presence of electric field reaches nearly value of zero field viscosity. Larger effect is observed for suspension of sample PPy2.

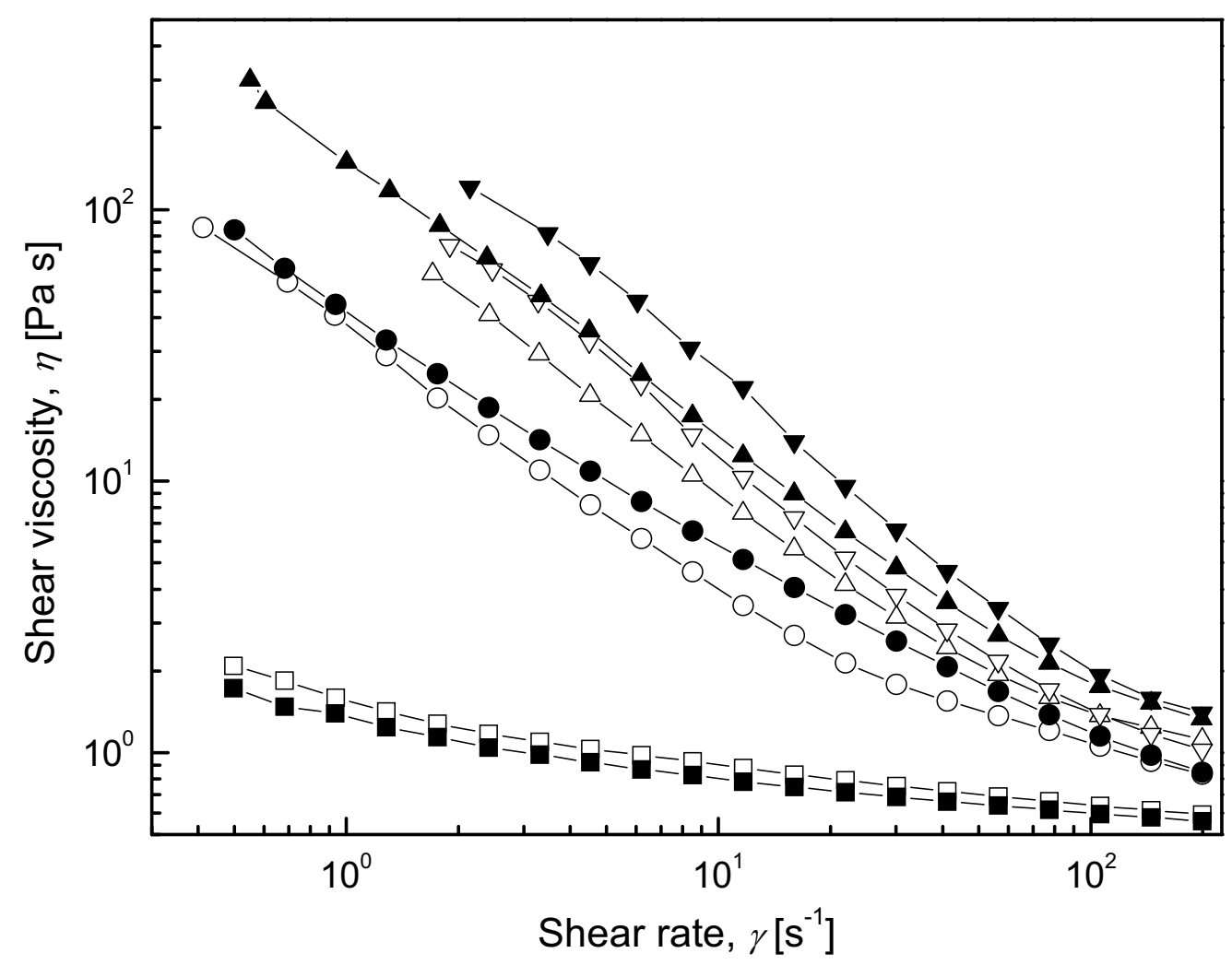

FIGURE 4. Dependence of viscosity, $\eta$, on shear rate, $\gamma$, for 3 wt. \% polypyrrole ribbon particles PPy1 (open) and

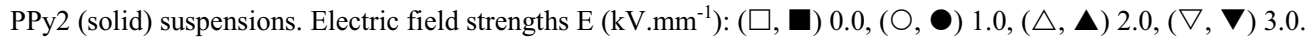


Figure 5 shows the effect of electric field on viscoelastic properties of PPy1 and PPy2 sample suspensions. Elastic modulus $G^{\prime}$ increases more than 3 orders of magnitude in the presence of electric field. Independency of $G^{\prime}$ of frequency suggests the existence of a stiff and elastic structure induced by the electric field.

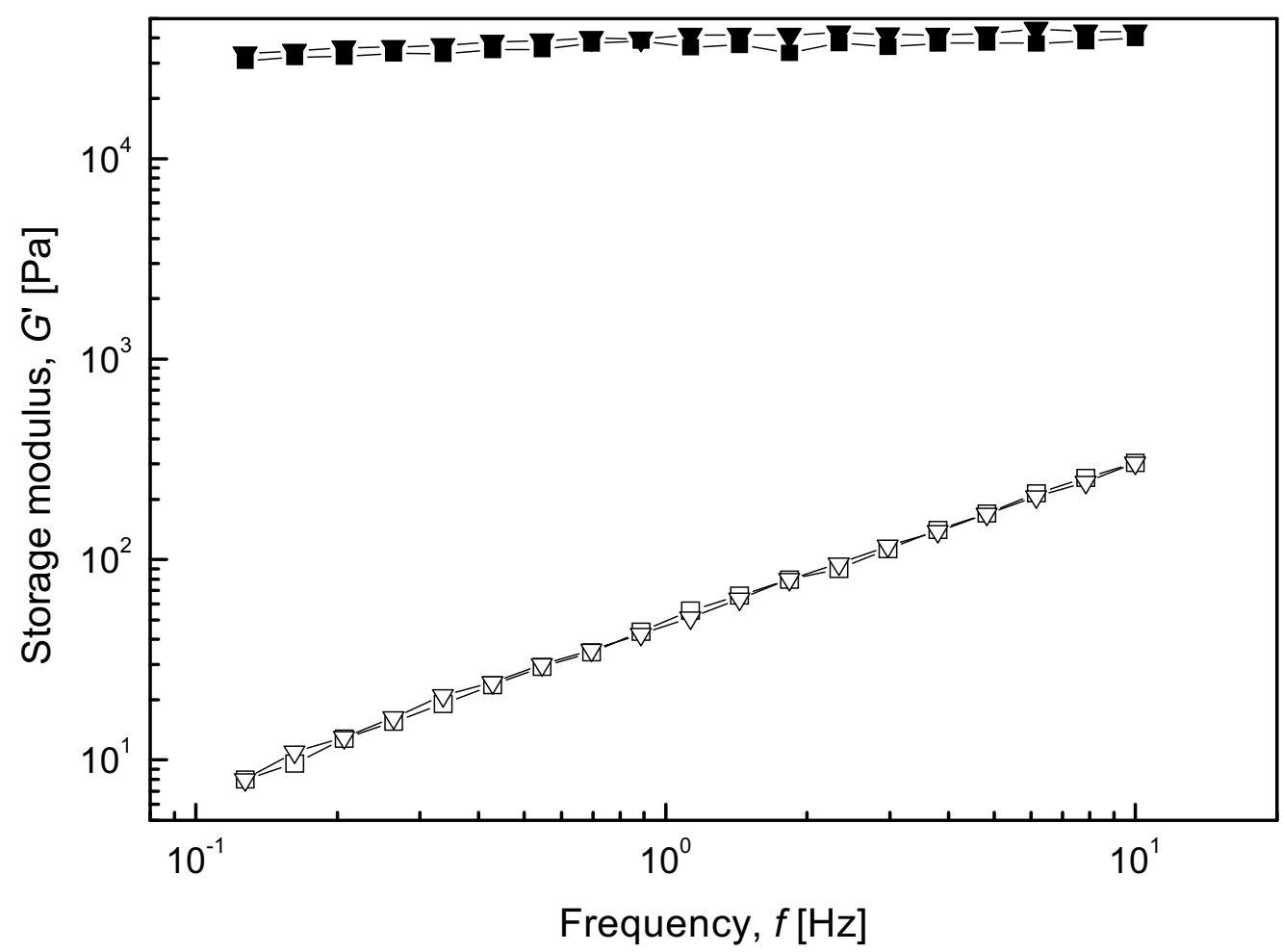

FIGURE 5. The dependence of storage modulus, $G$ ', on the frequency, $f$, for 3 wt. \% polypyrrole ribbon particles PPy1 $(\square, \boldsymbol{\square})$ and PPy2 $(\nabla, \boldsymbol{\nabla})$ suspension. Electric field strengths E (kV/mm): 0 (open), 3 (solid).

Different ER behavior is reflected in different dielectric properties of suspensions of samples PPy1 and PPy2. Figure 6 shows relative permittivity $\varepsilon$ ' and dielectric loss factor $\varepsilon$ ' as function of electric field frequency. Suspension of sample PPy2 shows shift of dielectric loss factor maximum to higher frequencies comparing to sample PPy1. It means that relaxation time decreases. Furthermore values of relative permittivity as well as polarizability of particles (defined as difference between relative permittivities at zero and infinite frequency) are higher for suspension of PPy2 sample particles. Therefore 
stronger ER effect (higher yield stress, higher storage modulus, higher viscosity) was observed for PPy2 sample suspension.

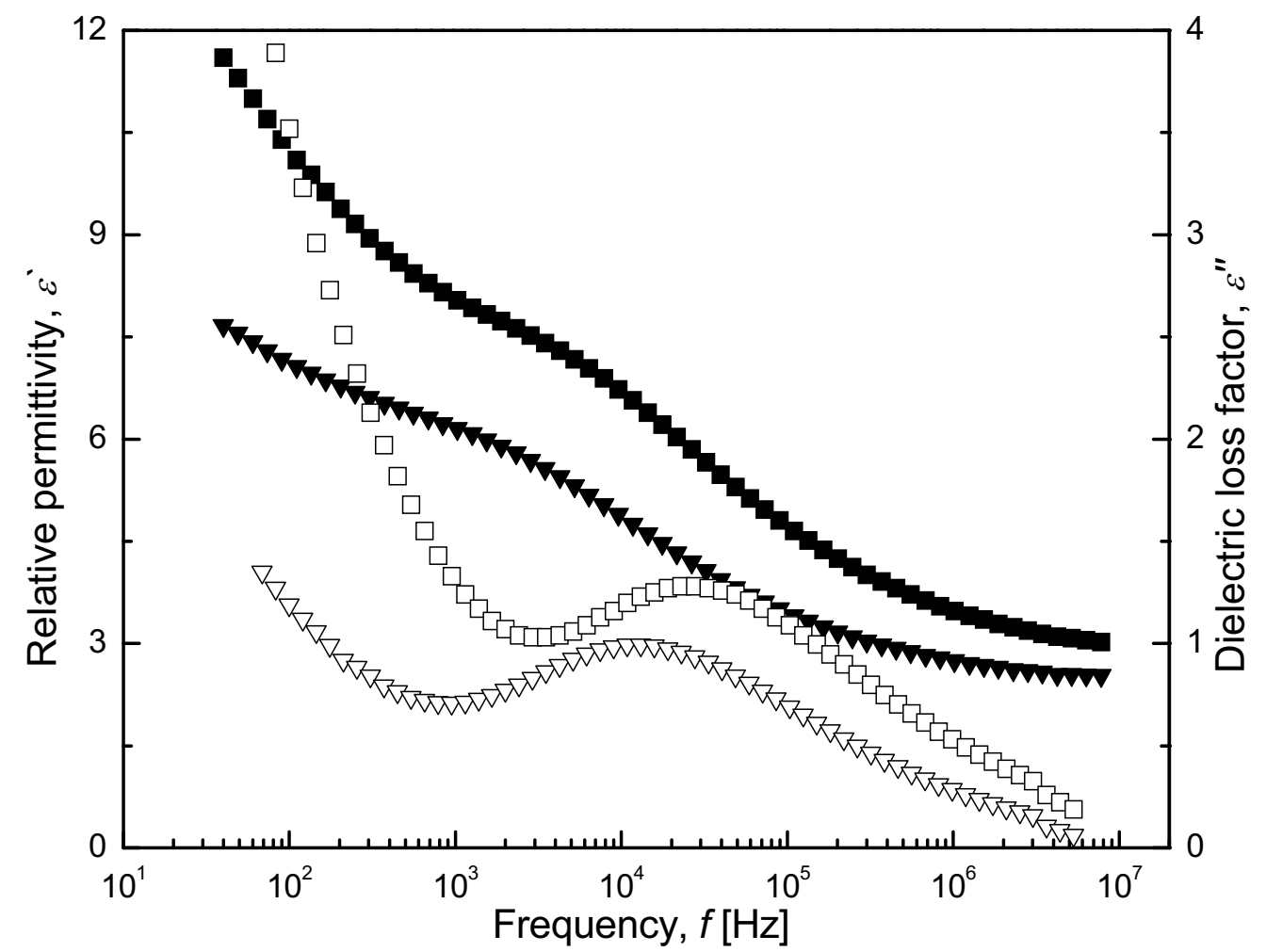

FIGURE 6. Dependence of relative permittivity, $\varepsilon$ ', (solid) and dielectric loss factor, $\varepsilon$ ', (open) on frequency, $f$, for 3 wt. \% polypyrrole ribbon particles $\operatorname{PPy} 1(\square, \boldsymbol{\square})$ and $\operatorname{PPy} 2(\nabla, \boldsymbol{\nabla})$ suspensions.

\section{CONCLUSION}

The ER behavior of novel PPy ribbon particle suspensions in silicone oil was investigated. PPy sample particles with different conductivity were synthesized. It was shown that only $3 \mathrm{wt}$ \% suspensions of PPy ribbon particles exhibit very large ER effect. Intensity of ER effect is strongly influenced by particle morphology as well as by suitable dielectric properties. 


\section{ACKNOWLEDGMENTS}

The authors wish to thank to the Grant Agency of the Czech Republic and the Ministry of Education, Youth and Sports of the Czech Republic for the financial support of Grant No. 202/09/1626 and MSM 7088352101, respectively.

This article was written with support of Operational Program Research and Development for Innovations co-funded by the European Regional Development Fund (ERDF) and national budget of Czech Republic, within the framework of project Centre of Polymer Systems (reg. number: CZ.1.05/2.1.00/03.0111).

\section{REFERENCES}

1. J .Wei, L. Zhao, S. Peng, J. Shi and Z. Liu, J. Sol-Gel. Sci. Techn. 47, 311-315 (2008).

2. U-S. Choi and B-G Ahn, Colloid Surface A 168, 71-76 (2000).

3. B. Sari, N. Yavas, M. Makulogullari, O. Erol and H. I. Unal, Reactive. Funct. Polym. 69, 808-815 (2009).

4. Y. Hirose and Y. Otsubo, Colloid Surface A 317, 438-442 (2008).

5. I. S. Lee, M. S. Cho and H. J. Choi, Polymer 15, 1317-1321 (2005).

6. A. Lengalova, V. Pavlinek, P. Saha, O. Quadrat and J. Stejskal, Colloid Surface A 227, 1-8 (2003).

7. H. Ahmari and S. Gh. Etemad, Rheol. Acta 48, 217-220 (2009).

8. Y. D. Liu, F. F. Fang and H. J. Choi, Mater. Lett. 64, 154-156 (2010).

9. I. S. Lee, J. Y. Lee, J. H. Sung and H.J.Choi, Synthetic Met. 152, 173-176 (2005).

10. Y. D. Kim and C. Song, J. Mater. Sci. 37, 5051-5055 (2002).

11. Q. Cheng, V. Pavlinek, A. Lengalova, C, Li, Y. He and P. Saha, Micropor. Mesopor. Mat. 93, 263- 269 (2006).

12. F. F. Fang, H. J. Choi and W-S. Ahn, Micropor. Mesopor. Mat. 130, 338-343 (2010).

13. F. F. Fang, M. S. Cho, H. j. Choi. S. S. Yoon and W-S Ahn, J. Ind. Eng. Chem. 14, 18-21 (2008).

14. Q. Cheng. Y. He. V. Pavlinek. A. Lengalova and P. Saha, J. Mater. Sci. 41, 5047-5049 (2006).

15. D. J. Yoon, Y. D. Kim, J. Colloid. Interf. Sci. 303, 573-578 (2006).

16. Y. D. Kim, J. H. Kim, Synthetic Met. 158, 479-483 (2008).

17. X. Zhang, J. Zhang, W. Song and Z. Liu, J. Phys. Chem. B, 110, 1158-1165 (2006).

18. C. H. Cho, H. J. Choi, J. W. Kim and M. S. Jhon, J. Mater. Sci. 39, 1883-1885 (2004).

19. L. C. Davis, J. Appl. Phys. 81, 1985-1991 (1997) 
Copyright of AIP Conference Proceedings is the property of American Institute of Physics and its content may not be copied or emailed to multiple sites or posted to a listserv without the copyright holder's express written permission. However, users may print, download, or email articles for individual use. 